\title{
Tarea Cognitiva de Detección y Corrección de Errores en la Comprensión de Textos Narrativos en Niños de Básica Primaria*
}

\section{Cognitive Detection and Error Correction Task in Narrative Text Comprehension in Elementary School Children}

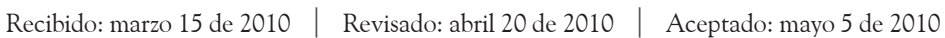

\author{
Diana Carolina Latorre VelásQUez ** \\ Universidad Pedagógica y Tecnológica de Colombia, \\ Tunja, Colombia \\ Hugo EsCOBAR-MELO *** \\ Pontificia Universidad Javeriana, Colombia
}

Para citar este artículo. Latorre Velásquez, D.C. \& Escobar-Melo, H. (2010). Tarea Cognitiva de Detección y Corrección de Errores en la comprensión de Textos Narrativos en Niños de Básica Primaria. Universitas Psychologica, 9 (3), 863-878.

\footnotetext{
* Artículo de investigación.

** Investigadora grupo desarrollo humano cognición y educación - UPTC.E-mail: cayollas@gmail.com

**** Facultad de Psicología, Edificio 95, Manuel Briceño S. J. Carrera 5 № 39-00. Tel. (57-1) 3208320 ext. 5757. Bogotá DC, Colombia. E-mail: escobarh@ javeriana.edu.co
}

\section{RES U MEN}

El presente artículo tiene como propósito fundamental presentar la construcción y desarrollo de una tarea cognitiva ubicada en el campo problemático de la comprensión del lenguaje, la cual constituye una alternativa metodológica para capturar el proceso de autorregulación, durante la lectura de un texto narrativo. Desde la perspectiva funcional, la tarea induce a la detección y corrección de errores semánticos, sintácticos y pragmáticos por parte de quien la ejecuta. La tarea, con gran fortaleza psicométrica (Alfa de Cronbach .820), inscrita en el paradigma de la contradicción, surgió del interés por construir herramientas de medición válidas y confiables que constituyan además alternativas metodológicas para el desarrollo de habilidades cognitivas y metacognitivas, cruciales en la habilidad de comprensión de textos narrativos.

Palabras clave autores

Desarrollo (SC 13830); Autorregulación (SC 23180); Metacognición (SC 31040);

Comprensión de lectura (SC 43110).

Palabras clave descriptor

Comprensión de lectura, metacognición, autorrealización (psicología).

\section{A B S T R A C T}

This paper aims to present the construction and development of a cognitive task located in the problematic area of language understanding, which is an alternative methodology to capture the self-regulatory process during the reading of a narrative text. From the functional perspective, the task leads to the detection and correction of errors semantic, syntactic and pragmatic on the part of whoever is running. The psychometric task with great fortitude (Cronbach's alpha .820) entered in the paradigm of contradiction arose from the interest in building tools valid and reliable measurement also constitute alternative methodologies for the development of cognitive skills and metacognitive skills crucial in understanding narrative texts. Keywords authors

Development (SC 13830); Self-Regulation (SC 23180); Metacognition (SC 31040); Reading Comprehension (SC 43110)

Keywords plus

Reading Comprehension, Metacognition, Self-Realization (Psychology). 


\section{Introducción}

Cuando niños y niñas se enfrentan a un texto narrativo, aparece, en primer plano, el proceso de comprensión el cual implica el dominio semántico, sintáctico y pragmático del lenguaje, funciones solidarias de una representación general del texto apoyada por el proceso de autorregulación.

Con la intención de dar cuenta de la autorregulación como pivote central de la comprensión lectora, se construyó y desarrolló una tarea cognitiva de detección y corrección de errores semánticos, sintácticos y pragmáticos por parte de quien la ejecuta (Latorre \& Escobar, 2009; Latorre, Ramírez \& Navarro, 2007).

Dicha tarea, con gran fortaleza psicométrica (Alfa de Cronbach .820), surgió de la necesidad e interés por construir herramientas de medición válidas y confiables que constituyan además verdaderas alternativas metodológicas para el desarrollo de habilidades tanto cognitivas como metacognitivas, en el marco de la cooperación interdisciplinaria entre la Psicología Educativa y la Pedagogía, cuando se enfrentan con los problemas escolares relativos al déficit comprensivo y representacional en la lectura de textos narrativos.

El proceso de construcción implicó varias fases tanto de pilotaje como de aplicación a muestras intencionales no probabilísticas, que cumplían con las características para las cuales fue desarrollada la tarea. Se mostrarán los hallazgos y consecuencias psicológicas y pedagógicas respectivas.

\section{Antecedentes Teóricos y empíricos}

La metodología de detección de errores, también conocida como el paradigma de la contradicción, permite abordar la habilidad comprensiva del lector de textos narrativos, a través de sus acciones de monitoreo y control de corte autorregulador, las cuales se despliegan tanto en la identificación de dichas fallas como en su corrección (Hacker, 1988; Winograd \& Johnston, 1980).

Los estudios reportados en la literatura evidencian las dificultades que, independientemente de la edad y el nivel académico, presentan los sujetos para detectar, y en consecuencia para corregir, los errores que se encuentran al leer un texto narrativo, que sin duda afectan su comprensión (Baker, 1984; Otero, 1998; Irrazabal, 2007). Dichas investigaciones evalúan la habilidad para detectar y corregir errores, comparando sujetos considerados como buenos o malos comprendedores (Chou \& Borchardt, 1985; Garner, 1980). Ponen de relieve el análisis de las causas frente a la dificultad en dicha habilidad que muestran en general los lectores (Matéus, 2007; Otero, 1998; Larigauderie, Gaonac'h \& Lacroix, 1998), al concluir que los que poseen mayor habilidad para comprender un texto, obtienen mayores rendimientos a la hora de detectar y corregir inconsistencias, frente a los que no lo hacen.

La habilidad de autorregulación que implica el monitoreo, la detección y precorrección de errores, el tipo de error insertado en el texto narrativo (Reis \& Spekman, 1983), la información dada a los sujetos frente a la presencia del error, la tipología textual, la capacidad o competencia de lectura alcanzada (Englert, Hiebert \& Stewart, 1988) y las demandas cognitivas (Beal, Bonitatibus \& Garrod, 1990), son, entre otros, excelentes indicadores respecto de los factores que influyen en la habilidad para capturar el proceso de comprensión.

Dentro de las exigencias cognitivas que demanda la detección y corrección de errores en un texto, se encuentra la memoria de trabajo (Hacker, Plumb, Butterreld, Quathamer \& Heineken, 1994; Levy \& Begin, 1984) la cual, según Baddley (1986), involucra tres subcomponentes: el bucle fonológico, el sistema ejecutivo central y la agenda visoespacial. Dichos elementos hacen que los errores, especialmente de carácter semántico y sintáctico, sean de difícil detección, en comparación con los errores de tipo ortográfico y tipográfico.

Dentro de los experimentos realizados por Larigauderie, Gaonac'h y Lacroix (1998), en los que se controló la memoria de trabajo especialmente lo relacionado con el subcomponente del bucle fonológico y el sistema ejecutivo de control, se concluyó que los sujetos mejoran el rendimiento frente a su habilidad de detección y corrección independientemente de la tipología de error, y 
mejoran especialmente la capacidad para detectar errores de carácter semántico y sintáctico. Dicha competencia de detección implica la retención temporal de información, que facilita la comprensión del segmento del texto que contiene el error, con el fin de establecer las relaciones de significado con anteriores pasajes.

Otros factores pueden modificar las demandas cognitivas involucradas en una tarea de detección de errores; algunos de éstos son los objetivos que se establecen frente al texto, la profundidad de la revisión, la orientación del sujeto hacia la comprensión del texto (Beal, Bonitatibus \& Garrod, 1990) y las acciones de monitoreo y control, como la relectura, que pueden conducir al aumento o disminución de la tasa de detección de errores, especialmente de carácter semántico y sintáctico (Levy, Newell, Snyder \& Timmins, 1986).

Dentro de los estudios que resaltan la detección de errores como una forma de aproximarse al proceso metacognitivo en niños, se encuentran los de Jaramillo, Montaña y Rojas (2006) con niños colombianos de 9 y 10 años. En su propuesta, que solo contempla errores de tipo semántico, hallaron que los sujetos presentan dificultad cuando los errores se refieren a la insuficiencia de información. Lo anterior se atribuye más a variables de carácter contextual que a la capacidad de los sujetos en sentido estricto.

Vergara y Velásquez (1999) coinciden en afirmar que los niños de estas edades presentan correlaciones positivas entre la experiencia metacognitiva y la comprensión, a la hora de detectar errores de tipo léxico; sin embargo, señalan que se les dificulta la detección de errores semánticos y sintácticos.

Otero y Campanario (1990) realizaron un estudio para evaluar la forma en que diversos estudiantes regulaban la comprensión de textos en ciencias, logrando identificar tres grupos de sujetos. El primer grupo no detectaba las inconsistencias en el texto, el segundo grupo evaluaban la comprensión pero no eran capaces de regular, y un tercero, reali- zaba una buena evaluación del texto acompañada de regulación frente a la comprensión, función que los llevaba a tener una representación adecuada de lo leído.

Para Matéus (2007) un sujeto que lee integra la información con sus propios conocimientos y tiene disponible, en términos cognitivos, lo que procesa más fácilmente, en comparación con un sujeto que simplemente suma información. En este caso, la memoria de trabajo sobrepasa su capacidad y no permite establecer relaciones entre la información y la habilidad para detectar errores.

La detección de inconsistencias se ha investigado de varias maneras, a través de reportes verbales, después de la lectura en la que se solicita a los lectores que subrayen los problemas encontrados en el texto. Por medio del registro de relecturas y tiempos de lectura en los fragmentos que se suponen problemáticos, la mayoría de los estudios ha demostrado que tanto jóvenes como adultos tienen dificultades para hallar inconsistencias en la lectura (Otero, 1998).

Con el diseño y construcción de la tarea cognitiva, que se presenta en este artículo, se propone una herramienta metodológica que privilegia el conocimiento procedural como alternativa que permite la recuperación de las acciones, que evidencian que el niño piensa en lo que hace, al cumplir el objetivo de detectar, identificar y corregir errores en un texto, y proceso inherente a la comprensión del mismo.

El diseño de la tarea recupera los resultados de los estudios anteriormente mencionados al controlar la memoria de trabajo, advertir a los niños sobre la presencia del error a través de la instrucción dada, además de trabajar sobre una estructura textual familiar para la población. El diseño de la tarea capaz de capturar la actividad cognitiva de niños y niñas, implicó un reto metodológico, en términos del análisis lógico, psicológico y procedimental, además de un procedimiento psicométrico frente al proceso de detección -identificación del error- con el fin de otorgarle mayor rigurosidad al resultado que aquí se presenta. 


\section{Método}

\section{Descripción de la tarea}

Para el diseño de la tarea cognitiva, se recurrió a la metodología de detección de errores de Garner (1980), la cual permite dar cuenta del proceso de comprensión de lectura de forma general, además de observar las acciones de monitoreo y control en términos de si los niños y niñas detectan e identifican el error, lo corrigen y cómo lo corrigen.

La tarea cognitiva se caracteriza por instalarse en el dominio del lenguaje, específicamente en la lectura. La tipología textual que se utilizó fue el texto narrativo, frente al cual los niños y niñas escolares están familiarizados. Para resolverla, es necesario tener una competencia lectora, razón por la cual, al diseñarla, se tiene presente que evidentemente el niño debe poseer cierto vocabulario y tener conocimientos gramaticales.

Lo que se resalta en la tarea cognitiva es precisamente el hecho de que el niño de básica primaria, al detectar errores en la lectura, realiza acciones que dan cuenta del proceso de comprensión implicado, lo que constituye un monitoreo que conlleva a establecer relaciones entre el conocimiento, la acción y las demandas del texto; de esta forma la tarea se convierte en la elicitadora del proceso (Puche-Navarro, 2008).

Se privilegia el conocimiento procesual, sobre el declarativo, ya que la tarea cognitiva exige la realización de acciones que evidencian que el niño está pensando sobre lo que está haciendo, tales como releer párrafos, todo el fragmento, centrar la atención en partes del texto, borrar y cambiar su respuesta. La detección de errores se suscita a partir de la tarea, por lo tanto no se busca que el niño, de manera verbal, reporte su conocimiento gramatical o dé cuenta verbalmente de cómo hace para resolver la tarea o alcanzar el objetivo que se le plantea.

Controla la memoria de trabajo, al presentar la información a través de dos canales de entrada de la información, visual y auditivo, donde la razón de dicho control se toma de Larigauderie, Gaonac'h y Lacroix (1998), quienes sugieren, como muy importante, el control de este tipo de memoria para mejorar la tasa de detección de errores en un texto. La tarea presenta un texto narrativo sobre temas cotidianos acerca de vivencias de los niños, la idea del texto es producto de una recolección de cuentos construidos por niños de grado tercero, y parte de la tarea se presenta combinando imagen y texto, todo esto con el fin de capturar el interés de los niños. A continuación, se presenta la tarea cognitiva teniendo en cuenta, su descripción, las restricciones, instrucciones y demandas cognitivas.

La tarea cognitiva se estructura en el contexto de un texto narrativo impreso a doble espacio que incluye errores que dificultan su comprensión, construido originalmente por Latorre, Ramírez y Navarro (2007) y adaptado para esta versión, el cual contiene tres fragmentos que presentan una idea principal, donde cada fragmento conserva la estructura narrativa de inicio, nudo y desenlace, acompañada de imagen. La extensión de cada fragmento no sobrepasa las 210 palabras y el texto se acompaña de audio; el experimentador utiliza un registro de observación de acciones.

El texto utilizado incluye 18 errores, seis por cada fragmento pertenecientes a tres tipologías de error: semántica, que afecta el significado local o global del texto como el cambio del final y la inclusión de palabras que afectan la coherencia de un párrafo; sintáctica, que afecta la estructura del sintagma (global) y local cuando se refieren a un cambio en parte de la estructura, como un pronombre o el uso inadecuado de plural/singular; y, pragmática, que afecta la intención general del texto o intenciones particulares dentro de un párrafo, por lo cual se pueden generar transformaciones de creencias o transposición de deseos.

\section{Aplicación de la tarea en dos momentos}

En un primer momento, se presentan los textos con el fin de familiarizar al niño con el contenido, mostrándose primero el texto con sus imágenes y luego el sonido, para controlar la memoria de trabajo y el canal de entrada de la información. En un segundo momento, se le suministra a cada niño el texto para la detección, identificación y corrección de errores como una prueba de lápiz y papel. La aplicación 
de la tarea se realiza de forma individual, se dan las instrucciones por escrito y de forma verbal para garantizar que el niño comprenda lo que tiene que hacer e interiorice el objetivo de la tarea.

\section{Instrucciones de la tarea}

Primer momento: a) "Lee atentamente el siguiente cuento, cuando finalices de leer avisa al aplicador". b) "Escucha atentamente el cuento, cuando finalices de escucharlo avisa al aplicador".

Segundo momento: "A continuación te mostraremos nuevamente el cuento: 'Camilo y sus mascotas', pero esta vez el cuento tiene errores, que no permiten comprenderlo, tu objetivo consiste en subrayar todos los errores que puedas encontrar y corregirlos". En la Tabla 2, se presenta el texto utilizado en la tarea con los errores insertados.

Restricciones de la tarea: solo se debe subrayar lo que el niño determine como error.

\section{Funcionalidad}

Funcionalmente, la tarea posee un registro observacional (véase Apéndice $A$ ), en el cual se registran los datos sociodemográficos de los niños, tales

TABLA 2

Texto de la tarea "Camilo y sus Mascotas" con errores insertados

\begin{tabular}{|c|c|c|}
\hline & sh” & \\
\hline $\begin{array}{l}\text { aminando con mis pa- } \\
\text { eo-tienda, cuando una } \\
\text { s de mascotas y decidi- } \\
\text { ado en ella, pedí a mis } \\
\text { a insistente que me } \\
\text { ndió: "te la compra- } \\
\text { y te responsabilizas de } \\
\text { r una de las vitrinas me } \\
\text { pequeña bola de pelos } \\
\text { te a la dueña de la tien- } \\
\text { que está enrollado de } \\
\text { clla me respondió: "es un } \\
\text { imos venderlo y llevarlo } \\
\text { organicé un lugar en mi } \\
\text { la bola de pelos blancos } \\
\text { lerlín. En la primera } \\
\text { nté según las indicacio- } \\
\text { ienda de mascotas, a la } \\
\text {, mi padre me destruyó } \\
\text { ue tanta mi emoción, } \\
\text { se momento comencé a }\end{array}$ & $\begin{array}{l}\text { El fin de semana llegó de visita } \\
\text { mi tía clarita, quien se enteró } \\
\text { de la muerte de Merlín. Ella } \\
\text { queriendo recordarme lo sucedi- } \\
\text { do, me regaló un pez. } \\
\text { La tía Clarita me entregó el pez, } \\
\text { diciéndome: "debes bien cuidarlo } \\
\text { y alimentarlo". A lo que le res- } \\
\text { pondí: "no te preocupes tía, lo } \\
\text { haré todo al pie de la letra". } \\
\text { Recordando lo que me entregó } \\
\text { mi tía y antes de irme para el } \\
\text { colegio le di mucha, mucha } \\
\text { comida a flash, porque así no } \\
\text { moriría de hambre como le paso } \\
\text { a Merlín. Lo que nunca ima- } \\
\text { giné, era que de tanto comer } \\
\text { se fue a enfermar y tendría que } \\
\text { cuidarlo. } \\
\text { Pasadas dos semanas, después } \\
\text { de llegar del colegio, fui en } \\
\text { busca de Flash y lo ví diferente, } \\
\text { nadaba lento y estaba cada vez } \\
\text { más gordo, parecía un globo; } \\
\text { corrí en busca de mi padre para } \\
\text { que me ayudara, cuando regre- } \\
\text { samos lo encontramos contento } \\
\text { y comiendo. }\end{array}$ & $\begin{array}{l}\text { El fin de semana salí de paseo al parque } \\
\text { con mis padres, al llegar a la esquina del } \\
\text { semáforo bajé las manos y vi un pequeño } \\
\text { perro sucio de color café, que parecía } \\
\text { estar abandonado. } \\
\text { Le pedí a mis padres que lo lleváramos a } \\
\text { casa para asearlo y darle de comer y ellos } \\
\text { me dijeron: “ino! Olvida lo sucedido con } \\
\text { Merlín y Flash”. Llore y prometí cuidarlo } \\
\text { algunas veces, entonces mis padres me } \\
\text { dieron otra oportunidad, y llevé el perro } \\
\text { a casa. } \\
\text { Al llegar a casa, mis padres yo y bañamos } \\
\text { al perro y lo llamamos Nerón. Quedó } \\
\text { como nuevo, paso de color café a gris, y } \\
\text { sus ojos se veían aún más. Esta vez tuve } \\
\text { cuidado y lo alimenté adecuadamente, } \\
\text { solo con comida especial para perros dos } \\
\text { veces al día y mucha agua. } \\
\text { Al mes de tener a Nerón me encontraba } \\
\text { jugando con ella en el garaje de mi casa, } \\
\text { en ese momento mi mamá me llamó, } \\
\text { fui en su búsqueda, pero olvide cerrar la } \\
\text { puerta, descuido que aprovechó Nerón } \\
\text { para salir. } \\
\text { Cuando regresé a seguir jugando con } \\
\text { Nerón me di cuenta que el carro de la pe- } \\
\text { rrera municipal se lo estaba llevando, ioh } \\
\text { no!, Nerón va a estar con otros per }\end{array}$ \\
\hline
\end{tabular}

Nota. Se aclara que a los niños y niñas se les presentaron los fragmentos en hojas individuales y sin resaltar los errores, en este texto se hace para guiar al lector.

Fuente: elaboración propia. 
como: nombres y apellidos, fecha de nacimiento, edad, además de capturar las acciones de monitoreo y control que realizan durante el desarrollo de la tarea.

Para un adecuado funcionamiento se requiere que el investigador no tenga una intervención directa durante la resolución, ya que su papel se limita a dar las instrucciones de la tarea y asegurar que el niño comprenda el objetivo de la misma, es decir que el niño comprenda lo que tiene que hacer. Se requiere además de un observador independiente que registre las acciones del niño durante su ejecución.

Los materiales necesarios para llevar a cabo la tarea son, un computador con el programa de audio (Winap, reproductor de Windows media o BS Player). Posterior a la presentación de los cuentos, se utiliza papel, lápiz, borrador para la realización de la segunda fase de la tarea. Dentro de los materiales también se incluye preferiblemente una cámara de video para registrar todas las ejecuciones de los niños con el fin de analizarlas posteriormente con mayor rigurosidad.

El sujeto, para dar cuenta de la autorregulación, debe realizar acciones de monitoreo y control como la relectura, centrar la atención en errores, etc., que lo conduzcan a la detección-identificación y corrección de los errores. Tanto el número de errores detectados-identificados, las acciones que el sujeto realice para alcanzar el objetivo, como la forma de corrección, son los criterios más importantes de operacionalización del concepto (véase Tabla 3).

La tarea implica, además de capturar el proceso de autorregulación, que los niños y niñas sean capaces (la decodificación del código escrito), además, de realizar inferencias frente a lo leído y establecer relaciones entre los diferentes fragmentos del o los cuentos presentados.

Uno de los procesos cognitivos que se encuentra involucrado y que es particularmente necesario controlar, es la memoria de trabajo; por esta razón, en la tarea se presentan, en la primera fase, los textos escritos, y luego en audio; de esta manera se ejerce control sobre los canales representacionales de entrada de la información (inputs).

Es necesario que durante la aplicación de la tarea el niño esté atento y, por supuesto, que comprenda lo que lee. Dentro de los procedimientos de acción que pueden darse, están: leer el texto, escuchar el texto, leer el texto con errores, señalar los errores (durante la lectura o posterior a la lectura) y corregir el error.

\section{TABLA 3}

Criterios de puntuación, niveles de comprensión de la tarea y puntuaciones de autorregulación

\begin{tabular}{|c|c|c|c|c|c|}
\hline $\begin{array}{l}\text { Nivel } \\
\text { de com- } \\
\text { prensión }\end{array}$ & $\begin{array}{l}\text { Definición nivel } \\
\text { de comprensión }\end{array}$ & $\begin{array}{c}\text { Criterios autorregulación } \\
\text { tarea }\end{array}$ & $\begin{array}{l}\text { Puntua- } \\
\text { ciones }\end{array}$ & $\begin{array}{l}\text { Número } \\
\text { de errores }\end{array}$ & $\begin{array}{c}\text { Ejecuciones (ejemplo): } \\
\text { Acciones que pueden realizar } \\
\text { los sujetos }\end{array}$ \\
\hline \multirow[t]{2}{*}{ I } & \multirow{2}{*}{$\begin{array}{l}\text { Se hace una repre- } \\
\text { sentación muy su- } \\
\text { perficial e inadecua- } \\
\text { da del texto al saber } \\
\text { que existen fallas en } \\
\text { él, pero no puede } \\
\text { detectarlas e identi- } \\
\text { ficarlas. }\end{array}$} & $\begin{array}{l}\text { Las diferentes acciones de } \\
\text { monitoreo y control que rea- } \\
\text { liza no le permiten detectar } \\
\text { e identificar las fallas dentro } \\
\text { del texto; sin embargo, repor- } \\
\text { ta falsos errores. }\end{array}$ & 1 & 0 & $\begin{array}{l}\text { El niño lee el texto de forma } \\
\text { global y luego subraya parte } \\
\text { del texto que no tiene erro- } \\
\text { res. }\end{array}$ \\
\hline & & $\begin{array}{l}\text { Las diferentes acciones de } \\
\text { monitoreo y control que } \\
\text { realiza le permiten detectar } \\
\text { fallas en las partes del texto } \\
\text { donde están los errores, pero } \\
\text { no los identifica. }\end{array}$ & 2 & 0 & $\begin{array}{l}\text { El niño se detiene en la lectu- } \\
\text { ra y subraya un párrafo o par- } \\
\text { te del texto, pero no señala el } \\
\text { error o falla; realiza acciones } \\
\text { como revisar el texto, o releer } \\
\text { no logra identifica el error. }\end{array}$ \\
\hline
\end{tabular}




\begin{tabular}{|c|c|c|c|c|c|}
\hline $\begin{array}{l}\text { Nivel } \\
\text { de com- } \\
\text { prensión }\end{array}$ & $\begin{array}{l}\text { Definición nivel } \\
\text { de comprensión }\end{array}$ & $\begin{array}{c}\text { Criterios autorregulación } \\
\text { tarea }\end{array}$ & $\begin{array}{l}\text { Puntua- } \\
\text { ciones }\end{array}$ & $\begin{array}{l}\text { Número } \\
\text { de errores }\end{array}$ & $\begin{array}{c}\text { Ejecuciones (ejemplo): } \\
\text { Acciones que pueden realizar } \\
\text { los sujetos }\end{array}$ \\
\hline \multirow[t]{2}{*}{ II } & \multirow[t]{2}{*}{$\begin{array}{l}\text { Se hace una repre- } \\
\text { sentación parcial del } \\
\text { texto al saber que } \\
\text { existen fallas en él y, } \\
\text { por lo tanto, puede } \\
\text { detectar, identificar } \\
\text { y corregir de } 1 \text {-2 } \\
\text { tipologías de error. }\end{array}$} & $\begin{array}{l}\text { Las diferentes acciones de } \\
\text { monitoreo y control que } \\
\text { realiza le permiten detectar e } \\
\text { identificar 1-2 tipologías de } \\
\text { error de forma parcial, propo- } \\
\text { ne correcciones relacionadas } \\
\text { con el texto o con el tipo de } \\
\text { error. } \\
1 \text { tipología de error total y } 1 \\
\text { parcial, propone correcciones } \\
\text { relacionadas con el texto o } \\
\text { con el tipo de error. }\end{array}$ & 3 & $1,2,3$ & $\begin{array}{l}\text { Lee y subraya simultánea- } \\
\text { mente algunos errores, por } \\
\text { ejemplo semánticos y prag- } \\
\text { máticos, pero solo locales. } \\
\text { Se centra en los errores ini- } \\
\text { cialmente identificados, que } \\
\text { no le permiten identificar y } \\
\text { corregir nuevos errores. }\end{array}$ \\
\hline & & $\begin{array}{l}\text { Las diferentes acciones de } \\
\text { monitoreo y control que } \\
\text { realiza le permiten detectar e } \\
\text { identificar } 1 \text { - } 2 \text { tipologías de } \\
\text { error de forma total, propone } \\
\text { correcciones relacionadas } \\
\text { con el texto o con el tipo de } \\
\text { error. }\end{array}$ & 4 & $2,3,4$ & $\begin{array}{l}\text { Relee párrafos y subraya erro- } \\
\text { res semánticos y pragmáticos } \\
\text { tanto locales como globales; } \\
\text { no cambia la acción de releer } \\
\text { para realizar la corrección. }\end{array}$ \\
\hline \multirow[t]{2}{*}{ III } & \multirow[t]{2}{*}{$\begin{array}{l}\text { Se hace una repre- } \\
\text { sentación total y } \\
\text { adecuada del texto } \\
\text { al saber que existen } \\
\text { fallas en él y, por lo } \\
\text { tanto, puede de- } \\
\text { tectar, identificar y } \\
\text { corregir } 3 \text { tipologías } \\
\text { de error. }\end{array}$} & $\begin{array}{l}\text { Las diferentes acciones de } \\
\text { monitoreo y control que } \\
\text { realiza le permiten detectar } \\
\text { e identificar } 3 \text { tipologías de } \\
\text { error de forma parcial, propo- } \\
\text { ne correcciones relacionadas } \\
\text { con el texto o con el tipo de } \\
\text { error. } \\
2 \text { tipos parcial y } 1 \text { total, pro- } \\
\text { pone correcciones relaciona- } \\
\text { das con el texto o con el tipo } \\
\text { de error. }\end{array}$ & 5 & 3,4 & $\begin{array}{l}\text { Lee, detecta, identifica y } \\
\text { corrige simultáneamente } \\
\text { errores semánticos, pragmáti- } \\
\text { cos y sintácticos tanto locales } \\
\text { como globales; reporta de } \\
\text { manera explícita información } \\
\text { que evoca de la memoria para } \\
\text { corregir los errores. }\end{array}$ \\
\hline & & $\begin{array}{l}\text { Las diferentes acciones de } \\
\text { monitoreo y control que } \\
\text { realiza le permiten detectar } \\
\text { e identificar } 3 \text { tipologías de } \\
\text { error de forma total, propone } \\
\text { correcciones relacionadas } \\
\text { con el texto o con el tipo de } \\
\text { error. } \\
2 \text { tipologías totales y } 1 \text { par- } \\
\text { cial, propone correcciones } \\
\text { relacionadas con el texto o } \\
\text { con el tipo de error. }\end{array}$ & 6 & 5,6 & $\begin{array}{l}\text { Lee, detecta, identifica y co- } \\
\text { rrige simultáneamente erro- } \\
\text { res semánticos, pragmáticos } \\
\text { y sintácticos tanto locales co- } \\
\text { mo globales; cuando corrige } \\
\text { relaciona ideas de diferentes } \\
\text { fragmentos del cuento. }\end{array}$ \\
\hline
\end{tabular}

Fuente: elaboración propia. 
Dentro de este procedimiento se presentan acciones como releer, centrar la atención en errores específicos que implican secuencias de estas acciones que son las que van a marcar las diferencias individuales de niños y niñas, al igual que el número de errores identificados y las correcciones realizadas.

\section{Participantes}

Niños y niñas de básica primaria de los grados $3^{\circ}, 4^{\circ}$ y $5^{\circ}$ de un colegio de la ciudad de Duitama (Departamento de Boyacá, Colombia). La muestra se seleccionó de manera intencional no probabilística, y se constituyó como "cautiva" en tanto en cuanto corresponde al universo institucional.

La muestra estuvo conformada para el estudio piloto por 20 niñas y niños de los grados tercero, cuarto y quinto. Para el estudio principal, se contó con la participación de 65 niñas y niños que corresponden a la totalidad de la población de los grados tercero, cuarto y quinto de básica primaria del colegio mencionado. La muestra se distribuyó en $50,8 \%$ pertenecientes al género femenino, el 49,2\% al género masculino; en cuanto al grado, se encuentra que el $29.2 \%$ pertenecen al grado tercero, el 30,8\% al grado cuarto y el 40\% al grado quinto. En cuanto a la distribución por edad, se encuentra que las edades oscilan de los ocho años cero meses a los 12 años dos meses.

El promedio de edad está en 9 años y 9 meses; el 15,2\% de los sujetos está en el intervalo de los 8 años cero meses a los 8 años y 9 meses; el 32,2\% de los 9 años cero meses a los 9 años y 9 meses; el $32,3 \%$ de los 10 años cero meses y los 10 años y 9 meses; los niños que tienen una edad superior a los 10 años, se distribuyen así: el 18,4\% entre los 11 años cero meses y los 11 años y cinco meses, y el 1,5\% tiene 12 años y 2 meses.

\section{Procedimiento de construcción de la tarea}

Fase I - Diseño de la tarea cognitiva

En el texto narrativo de la tarea "Camilo y sus mascotas" y "Valentina y Vanessa" de Latorre, Ramírez y Navarro (2007), se introdujeron errores de carácter semántico, sintáctico y pragmático de tipo local y global, además de construir cuatro versiones narrativas con los errores insertados. Se solicitó la colaboración de una Licenciada en Idiomas para la revisión de los tipos de errores insertados. Igualmente se establecieron los criterios iniciales de aplicación y calificación de la tarea.

Fase II - Prueba piloto

Se realizó una prueba piloto con 20 estudiantes que cumplía características similares a la muestra del estudio, con el fin de realizar ajustes a la tarea, a los criterios de puntuación y diseño de un registro de observación frente a las acciones de monitoreo y control, que realizan los sujetos participantes.

Fase III - Aplicación de la tarea cognitiva Una vez ajustada la tarea se procedió a aplicarla a una muestra intencional no probabilística de 65 niños y niñas. Para esta aplicación se entrenó a estudiantes del "Semillero mentes en acción" del Grupo de Investigación Desarrollo Humano Cognición y Educación de la Escuela de Psicología de la Universidad Pedagógica y Tecnológica de Colombia, para ser observadores del proceso y colaboradores en las aplicaciones y diligenciamiento del registro observacional.

\section{Resultados}

Los resultados de la aplicación piloto revelaron que la tarea cognitiva efectivamente permitía detectar e identificar las tres tipologías propuestas tanto a nivel local como global. La tipología que mejor fue detectada e identificada fue la semántica, y la que generó mayor dificultad, fue la sintáctica. Frente a la intención del pilotaje para seleccionar una versión de la tarea, se estableció la media total del error por fragmento de cada versión de cada cuento, encontrándose que el cuento que mejor se comportó fue "Camilo y sus mascotas - versión II".

Con el fin de darle mayor rigurosidad a la tarea diseñada, se realizó un análisis psicométrico tomando cada error insertado en el texto como si fuese un ítem, además de establecer una escala de calificación de 1 a 3, donde 1 corresponde a la 
opción "no detecta - identifica", 2 corresponde a "detecta", y 3 corresponde a "identifica".

Cuando un error no es subrayado por el sujeto, se calificó con una puntuación de 1; para el caso en que un sujeto subrayara alguna parte del texto donde estaba el error pero que la evidencia permitió concluir que no lo identifica con exactitud, se calificó con una puntuación de 2, y para el caso en que un sujeto subrayara con precisión el error, se le asignó la puntuación 3.

El nivel de confiabilidad de la tarea se calculó en un alfa de Cronbach de 0,82, lo que muestra que los diferentes errores introducidos en los textos son consistentes y homogéneos, además de observarse precisión en los datos, lo que se corrobora con el nivel de varianza y el error aleatorio bajo.

En la Tabla 4 se muestra el índice de confiabilidad de cada error (ítems), donde se observa que todos los ítems son consistentes y homogéneos, ninguno es inferior al índice general de la prueba y por lo tanto no es necesario descartar ninguno de los ítems incluidos.

La Tabla 5 muestra los intervalos de confianza al 95\%, el cual se obtuvo a través del coeficiente de correlación intraclase, los puntajes oscilan entre 0,750 y 0,878 que evidencian una confiabilidad significativa y están dentro del margen del coeficiente de confiabilidad general para la tarea.

\section{TABLA 4}

Estadísticas totales ítems de la tarea cognitiva

\begin{tabular}{lccccc}
\hline & $\begin{array}{c}\text { Scale Mean if } \\
\text { Item Deleted }\end{array}$ & $\begin{array}{c}\text { Scale Variance if } \\
\text { Item Deleted }\end{array}$ & $\begin{array}{c}\text { Corrected Item- } \\
\text { Total Correlation }\end{array}$ & $\begin{array}{c}\text { Squared Multiple } \\
\text { Correlation }\end{array}$ & $\begin{array}{c}\text { Cronbach's Alpha } \\
\text { if Item Deleted }\end{array}$ \\
\hline error1sint.g & 32,65 & 53,357 &, 294 &, 393 &, 817 \\
error2sint.l & 33,00 & 53,094 &, 454 &, 424 &, 810 \\
error3prag.local & 32,03 & 53,218 &, 256 &, 369 &, 821 \\
error4sem.local & 31,63 & 52,830 &, 420 &, 651 &, 811 \\
error5prag.g & 31,69 & 49,935 &, 610 &, 658 &, 800 \\
error6sem.g & 32,14 & 49,902 &, 487 &, 553 &, 806 \\
error7prag.l & 32,55 & 51,407 &, 407 &, 421 &, 811 \\
error8sint.g & 32,35 & 52,545 &, 301 &, 537 &, 818 \\
error9sem.l & 32,83 & 52,612 &, 367 &, 295 &, 813 \\
error10sint.1 & 32,74 & 51,884 &, 417 &, 491 &, 811 \\
error11prag.g & 32,98 & 55,953 &, 131 &, 356 &, 823 \\
error12sem.g & 31,60 & 51,244 &, 570 &, 686 &, 804 \\
error13sem.l & 32,51 & 51,629 &, 386 &, 433 &, 813 \\
error14prag.l & 32,91 & 53,460 &, 343 &, 339 &, 815 \\
error15prag.g & 32,15 & 51,632 &, 367 &, 302 &, 806 \\
error16sint.g & 32,35 & 50,232 &, 480 &, 367 &, 504 \\
error17sint.l & 32,43 & 49,687 &, 493 &, 523 &, 54 \\
error18sem.g & 31,89 & 49,504 & & &, 802 \\
\hline
\end{tabular}

Fuente: elaboración propia. 
TABLA 5

Coeficiente de correlación intraclase

\begin{tabular}{lccccccc}
\hline & \multirow{2}{*}{$\begin{array}{c}\text { Correlación } \\
\text { intraclase }\end{array}$} & \multicolumn{2}{c}{$95 \%$ Intervalo de confianza } & \multicolumn{4}{c}{ Prueba $F$} \\
\cline { 3 - 8 } & & Límite inferior & Límite superior & Value & df1 & df2 & Sig \\
\hline Medidas únicas & $0,202^{\mathrm{b}}$ & 0,143 & 0,285 & 5,556 & 64 & 1088 & 0,000 \\
Medidas promedio & $0,820^{\mathrm{c}}$ & 0,750 & 0,878 & 5,556 & 64 & 1088 & 0,000 \\
\hline
\end{tabular}

Fuente: elaboración propia.

En la Tabla 6 se presentan los resultados del análisis factorial que sugiere la agrupación de cinco componentes y que, de acuerdo con las correlaciones de cada uno de los ítems, se pueden agrupar en tres que corresponden a los tres fragmentos que conforman el cuento "Camilo y sus Mascotas". Cada fragmento guarda la estructura del texto narrativo (inicio-nudo-desenlace), pero a su vez cada fragmento del cuento se encuentra conectado por una idea principal.

Los ítems que corresponden al fragmento 1 corresponderían al error 1 hasta el error 6 pertenecientes a los diferentes tipos de error propuestos para el presente trabajo (sintáctico, semántico, pragmático local y global); para el caso del fragmento 2 , se encuentra una dispersión de los ítems debido, posiblemente, a la complejidad del texto que constituirán la construcción del segundo fragmento resulta coincidente con el nudo de una narración que genera mayor carga cognitiva; y, para el caso del fragmento 3 , los ítems que corresponden son del 13 al 18, donde el ítem 13 muestra una mayor carga factorial en otro componente; sin embargo, su carga factorial en el componente de agrupación es alta y por esta razón se agrupa en el fragmento 3 .

Respecto del comportamiento de los sujetos ante la detección e identificación de errores, en la figura 1 se presenta la distribución de la muestra en los tres niveles de comprensión por fragmento, donde se observa que la muestra en los tres fragmentos se distribuye en los tres niveles de comprensión propuestos.

En los fragmentos 1 y 2 , el nivel de comprensión que predomina es el 2; es decir, que la mayoría de los sujetos se hacen una representación parcial
TABLA 6

Matriz de componentes

\begin{tabular}{|c|c|c|c|c|c|}
\hline & \multicolumn{5}{|c|}{ Componentes } \\
\hline & 1 & 2 & 3 & 4 & 5 \\
\hline error1sint.g & ,340 & ,445 &,- 443 & , 120 & ,336 \\
\hline error 2 sint.1 &, 524 & ,431 &,- 016 & ,099 &,- 186 \\
\hline error3prag.local & ,359 &,- 241 & ,086 &,- 638 & ,128 \\
\hline error4sem.local &, 562 &,- 519 &,- 261 &,- 083 &, 106 \\
\hline error5prag.g &, 721 &,- 359 &,- 086 &,- 235 &,- 031 \\
\hline error6sem.g & ,618 &,- 215 &,- 010 & ,349 &,- 362 \\
\hline error7prag.1 & ,478 & ,273 & ,514 & ,084 &,- 085 \\
\hline error8sint.g & ,377 & ,526 &,- 381 &,- 215 &,- 264 \\
\hline error9sem.1 & ,439 & ,101 &,- 058 & ,363 & ,495 \\
\hline error10sint.1 & ,491 & ,291 &,- 377 & , 100 &,- 196 \\
\hline error11prag.g & ,196 &,- 294 & ,293 & ,629 & ,190 \\
\hline error12sem.g & ,694 &,- 522 &,- 063 &,- 155 &,- 042 \\
\hline error13sem.1 & ,452 &, 273 & ,561 &,- 114 &,- 157 \\
\hline error14prag.l & ,398 & ,368 & ,130 &,- 427 & ,321 \\
\hline error15prag.g & ,456 &,- 224 &,- 184 &,- 002 & , 155 \\
\hline error16sint.g & ,563 & ,143 &,- 209 &, 228 & ,164 \\
\hline error17sint.1 & ,575 &, 160 & ,493 &,- 066 &, 272 \\
\hline error18sem.g & 656 &,- 066 & ,078 & 130 &,- 368 \\
\hline
\end{tabular}

Fuente: elaboración propia.

del texto al saber que existen fallas en él y, por lo tanto, pueden detectar, identificar y corregir de 1 a 2 tipologías de error. 
En el fragmento 3, el nivel que predomina es el 3; esto quiere decir que, a medida que avanza el desarrollo de la tarea, los sujetos mejoran sus desempeños lo que los conduce a tener una representación total del texto al saber que existen fallas en él y, por lo tanto, pueden detectar, identificar y corregir las 3 tipologías de error contempladas.

El análisis de varianza, a través de la prueba estadística $t$ para una muestra, evidencia diferencias significativas ( $t$ frag $1=24,7 ; g l=64 ; p<0,05$; tfrag2 $=27,5 ; g l=64 ; p<0,05 ;$ tfrag $3=22,9 ; g l$ $=64 ; p<0,05)$ entre los niveles de comprensión y los fragmentos $1(M=3,51 ; D E=1,34)$; fragmento $2(M=3,17 ; D E=1,12)$; y, fragmento 3 $(\mathrm{M}=3,69 ; \mathrm{DE}=1,62)$ de la tarea; por tanto, se acepta la hipótesis alterna frente a la existencia de diferencias significativas entre los niveles de comprensión de la tarea y los tres fragmentos, y se rechaza, en consecuencia, la hipótesis nula.

Lo anterior muestra que los niveles de comprensión varían entre los fragmentos, observándose que el fragmento que más nivel de comprensión presenta es el 3, del que se infiere que, a medida que el sujeto avanza en la tarea, va mejorando su habilidad de detectar, identificar y corregir los errores dentro de la lectura; es decir, de monitorear y controlar el proceso de lectura.

El análisis de varianza (ANOVA) entre los niveles de comprensión de la tarea por fragmento y el grado escolar, muestra que no existen diferencias significativas entre el nivel de comprensión de la tarea en el fragmento 1 y el grado $(F=0,119 ; P=$ 0,888); el nivel de comprensión de la tarea en el fragmento 2 y el grado $(F=0,507 ; P=0,605)$; , el nivel de comprensión del fragmento 3 y el grado $(F=1,579 ; P=0,214)$; esto conduce a aceptar la hipótesis nula frente a la existencia de diferencias por grado, y a rechazar la hipótesis alterna.

Esta ausencia de diferencias permite concluir que los desempeños de los sujetos de la muestra, frente al nivel de comprensión de la tarea, no dependen del grado académico o de estudio en el que se encuentre; es decir, esta variable no sería explicativa del proceso de comprensión.

Para el caso de la edad, el análisis de varianza no muestra diferencias significativas entre los niveles de comprensión de la tarea y la edad para el fragmento $1(F=0,1135 ; P=0,36)$ y $2(F=$

\section{Figura 1}

Distribución muestra por niveles de comprensión de la tarea

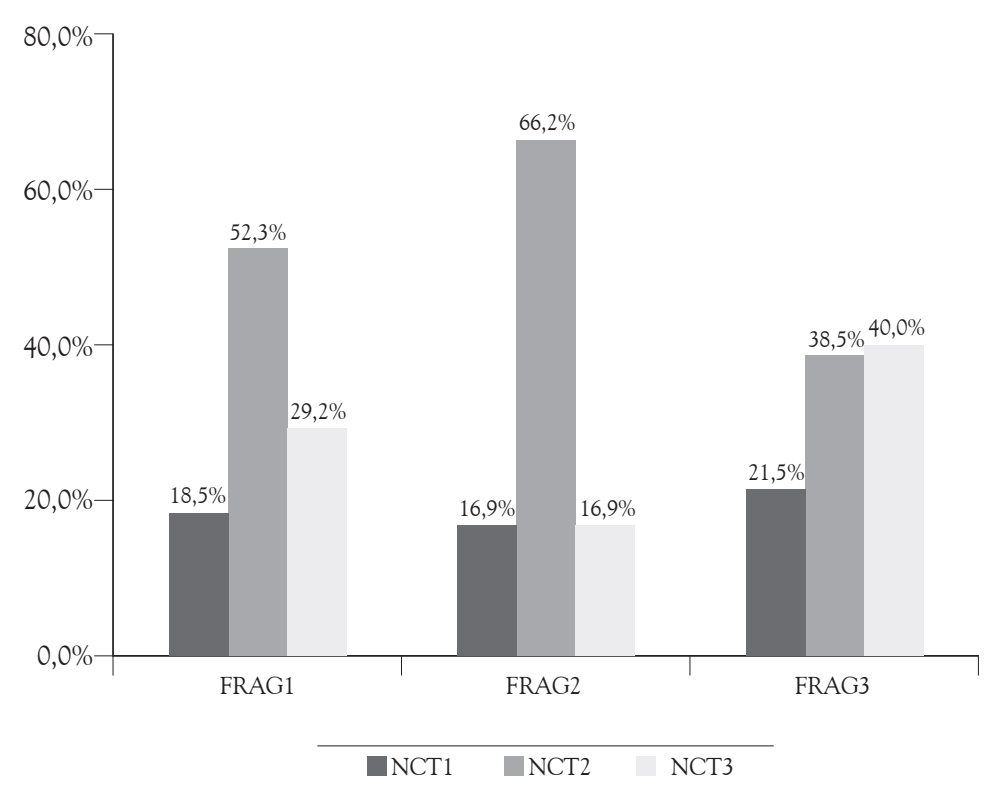

Fuente: elaboración propia. 
$1,42 ; P=0,16)$, tan solo se observa una diferencia significativa en el fragmento $3(F=2,84 ; P=$ $0,002)$. La diferencia se presenta en el intervalo de edad de $10(M=3)$ a $11(M=4,5)$ años, en la Tabla 7 se presentan los resultados de la prueba $t$ para muestras independientes, para el fragmento 3.

TABLA 7

Prueba t niveles de comprensión tarea y edad

\begin{tabular}{cccc}
\hline Edades & $T$ & $G l$ & Sig. (2 - tailed) $p<, 05$ \\
\hline $8-9$ & 0,000 & 18 & 1,00 \\
$9-10$ & 1,59 & 36 & 0,119 \\
$10-11$ & $-4,47$ & 41 & $0,000 *$ \\
$8-11$ & $-1,86$ & 23 & 0,075 \\
$9-11$ & $-1,95$ & 33 & 0,059 \\
\hline
\end{tabular}

Fuente: elaboración propia.

No se encuentran diferencias significativas entre el sexo y los niveles de comprensión fragmento $1(t=0,88 ; g l=63 ; p<0,05)$, fragmento $2(t=$ $0,000 ; g l=63 ; p<0,05)$ y fragmento $3(t=0,613$; $g l=63 ; p<0,05)$, razón por la cual, se acepta la hipótesis nula frente a la existencia de diferencias por sexo, y se rechaza la hipótesis alterna.

Frente al comportamiento de los sujetos en la detección e identificación de errores durante la tarea, se encuentra que los errores de mayor tasa de identificación son el semántico global, local y pragmático global, siendo el semántico global el que mejor comportamiento presenta.

Los errores que fueron menos detectados e identificados fueron los de tipo sintáctico local, global y pragmático local; el sintáctico local es el error que no detectan ni identifican en mayor proporción (véase Figura 2). Lo anterior es una evidencia que contradice a la mayoría de estudios en los que se ha implementado la técnica de detección de errores, los cuales afirman que el error semántico es el de mayor dificultad de detección, por la carga que ocasiona en la memoria de trabajo (Larigauderie, Gaonac'h \& Lacroix, 1998); este hallazgo puede atribuirse a la fase de control o fase 1 que conforma la tarea.

La Figura 3 muestra que, en los tres fragmentos, el error semántico global se comportó de igual

\section{FIGURA 2}

Porcentaje sujetos detección identificación de errores en la tarea

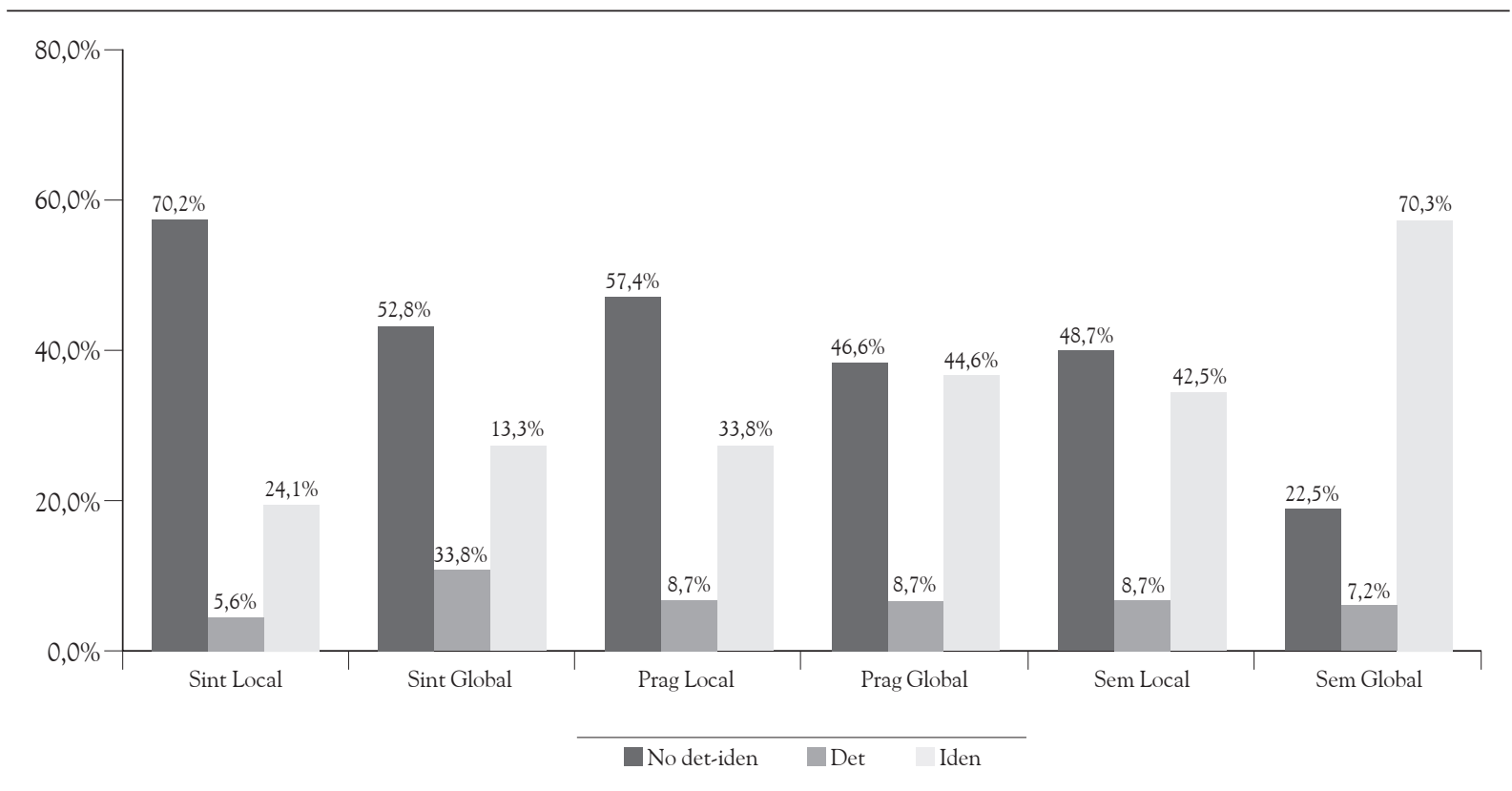

Fuente: elaboración propia. 
FIGURA 3

Moda por fragmento frente a la detección identificación de errores

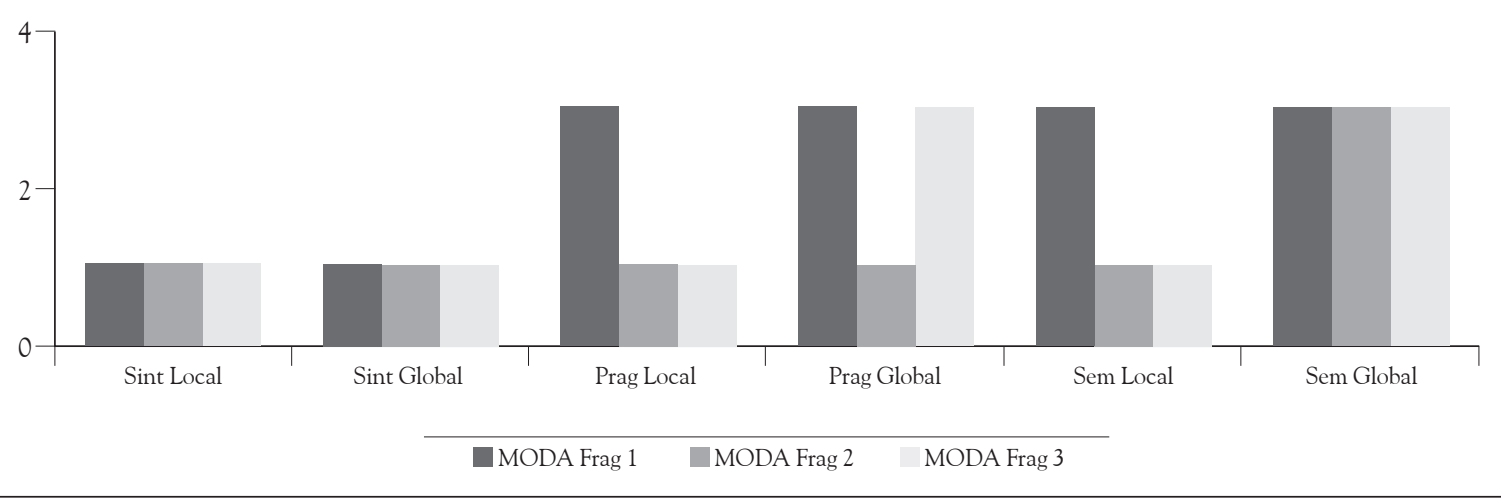

Fuente: elaboración propia.

manera, predominando una puntuación de tres asignada a la opción identifica el error; los errores sintáctico local y global predominaron para los tres fragmentos, la opción uno correspondiente a la opción no detecta-identifica el error; es decir, que estos errores no son fácilmente reconocidos por los sujetos a medida que avanza la lectura de cada fragmento.

Para el error pragmático local y semántico local en el fragmento 1, predomina la opción 3; en los fragmentos 2 y 3 disminuye el grado de identificación y predomina la opción 1 ; para el caso del error pragmático global, en el fragmento 1 predomina la opción 3, en el fragmento 2 disminuye a la opción $1, y$ vuelve a subir en el fragmento 3 a la opción 3 .

Se puede concluir, de los anteriores resultados, que cada tipología de error se comporta de manera diferente; el tipo de error que inicia en el fragmento 1 con una buena identificación, se mantiene así a lo largo de todos los fragmentos, al igual que el de menor identificación; sin embargo, errores de carácter local como el semántico y el pragmático que inician con una buena identificación, van disminuyendo al avanzar en el desarrollo de la tarea.

Lo anterior también muestra que, al ser el error de carácter semántico global el de mayor identificación, los sujetos comprenden que el significado principal del texto ha sido alterado, al cambiar el final de cada fragmento. Los errores sintácticos son los que mayor dificultad cognitiva implicaron. En la dimensión pragmática, se puede observar que los sujetos efectivamente reconocen la existencia de alteraciones frente a la intención que expresa el texto a nivel global y, por ende, local.

Por otro lado, frente a la detección de falsos errores, los sujetos atribuyeron que las anomalías contenidas en el texto eran de carácter ortográfico, llevándolos a proponer, en algunas ocasiones, falsas correcciones, mientras que aquellos que detectaban pero no identificaban el error, no proponían correcciones por no saber exactamente cuál era la anomalía que el texto contenía.

Se evidencia la relación entre distintos aspectos del comportamiento de los sujetos frente a la tarea, que conduce a la inferencia de que la habilidad para detectar y corregir los errores, puede estar relacionada con el tipo de interacción que cada sujeto asume frente al texto que lee y la representación que hace de éste, conclusión que va más allá de poseer una sencilla capacidad de descifrar el código escrito, y el conocimiento de reglas gramaticales.

\section{Discusión}

La tarea presentó un alto índice de confiabilidad ( $\alpha$ de Cronbach $=0,82$ ), aspecto que le da un valioso soporte, en función de la discriminación que hicieron los sujetos en cada fragmento de los ítems (errores) y frente a su habilidad para detectarlos 
e identificarlos en las condiciones e instrucciones que fueron impartidas para su resolución.

El análisis factorial realizado permitió observar que la tarea se comporta con buena validez, al permitir la agrupación de los ítems (errores) en los fragmentos contemplados; sin embargo, el fragmento 2 fue el único que presentó dispersión de ítems (errores), siendo éste el que mayor dificultad mostró, a pesar de que los tres fragmentos cuentan con las mismas características. En esta perspectiva, dicho fragmento parece generar una mayor exigencia cognitiva, por ser el que corresponde a la parte central (nudo) de la estructura de la narración (Oakhill \& Yuill, 1991).

El error semántico es el tipo de error de mejor comportamiento en la tarea, a pesar de que en varios estudios no sucede lo mismo como en el de Vergara y Velásquez (1999) y el caso del estudio colombiano realizado por Jaramillo, Montaña y Rojas (2006). El comportamiento de esta tipología de error en la tarea se atribuye a la fase inicial, donde se ejerce control de la memoria de trabajo, y al canal de entrada de la información al ser ésta una variable que afecta la habilidad para detectar errores en un texto (Hacker, 1994), especialmente de carácter semántico como lo exponen Larigauderie, Gaonac 'h y Lacroix (1998), en sus estudios pioneros donde a través de diferentes controles experimentales llevan a que el sujeto, efectivamente, aumente la tasa de identificación.

El error semántico es un error que permite evidenciar si los sujetos están haciendo una representación de significado del texto y, al ser el error de mayor tasa de identificación, estaría indicando una mayor apropiación frente a la idea principal de cada fragmento y del texto en general, y se relacionaría aún más con su comprensión; mientras que los errores sintácticos son los que presentaron mayor grado de dificultad para ser detectados, pese a que la población objeto de estudio posee ya un conocimiento de las normas sintácticas de su lengua materna.

La habilidad para detectar-identificar y corregir errores es considerada de difícil ejecución, el hecho de que los sujetos estén informados sobre la presencia del error y que conozcan previamente el texto conduce a facilitar el proceso de detección, sin convertirse en condiciones que lleven a los sujetos a comportarse con total éxito en la resolución de la tarea. Se observaron, en su mayoría, ejecuciones promedio, donde la tasa de detección-identificación y corrección del error osciló entre 2 a 4 errores por fragmento, evidenciándose apropiación del objetivo y de una comprensión parcial del texto.

La apropiación de los sujetos frente a la comprensión de la instrucción e incorporación del objetivo, se da en todo momento y en todos los sujetos; es así como algunos sujetos que estuvieron ubicados en el nivel 1 a pesar de no identificar errores, subrayaban partes del texto que no se consideraban errores "falsos errores", o inclusive fueron capaces de subrayar partes del texto donde efectivamente se había insertado un error, pero no lo podían identificar con precisión "detectaron, más no lo identificaron".

Las diferencias significativas entre fragmentos en el proceso de comprensión, se convierten en una evidencia de cómo este proceso se enriquece a lo largo del desarrollo de la tarea y hace que las acciones de monitoreo y control sean más eficaces, para incorporar en sus desempeños, las diferentes tipologías de error insertadas y tener una representación del texto completa y organizada, como lo sugiere Ordoñez (2000 citado por Gutiérrez, 2004), cuando afirma que es la acción del sujeto sobre el material la que produce en los niños diferentes niveles de abstracción o apropiación.

La tarea permitió elicitar acciones de monitoreo y control como la relectura, el centrarse en el error, detectar, identificar y corregir de manera simultánea, provocando en los sujetos la presentación de diferentes secuencias de acciones y mostrando que, efectivamente, la tarea permite la comprensión del proceso de autorregulación al conjugarse tres elementos: la detección de errores, la corrección de los mismos y las acciones que los sujetos realizan para detectarlos y corregirlos.

La combinación de acciones de autorregulación y su efectividad es un aspecto que cabe ser investigado en futuros estudios, en el dominio del lenguaje, con el fin de determinar la existencia de combinaciones de acciones que conduzcan a los 
niños y niñas a obtener altos desempeños en la comprensión de textos.

Lo anterior deja ver la fortaleza que tiene y el desafío metodológico que genera el trabajar procesos metacognitivos, basándose en la recuperación de lo que hace el sujeto, que evidencia en línea y permite, durante el desarrollo del proceso, la captura y análisis de información de manera sistemática (Spinillo \& Da Rocha, 2007), que sería imposible de recuperar con la misma precisión cuando se retoma desde la "opinión introspectiva" del sujeto luego de la realización de un test, o de haber resuelto algún problema.

\section{Referencias}

Baddeley, A. D. (1986). Working memory. Oxford: Oxford University Press.

Baker, L (1984). Children's effective use of multiple standards for evaluating their comprehension. Journal of educational Psychology, 76, 588-597.

Beal, C. R., Bonitatibus, G. J. \& Garrod, A. C. (1990). Fostering children's revision skills through training in comprehension monitoring. Journal of Educational Psychology, 82, 275-280.

Brown, A. (1980). Metacognitive development and reading. En R. J. Spiro, B. C. Bruce, W. F. Brewer (Eds.), Theoretical Issues in Reading comprehension (pp. 453-481). NJ: Lawrence Erlbaun.

Chou, V. \& Borchardt, K. M. (1985). Good and poor comprehenders' detection of errors revisited. Journal of Educational Research, 78 (4), 237-241.

Englert, C. S., Hiebert, E. H. \& Stewart, S. R. (1988). Detecting and correcting inconsistencies in the monitoring of expository prose. Journal of Educational Research, 81 (4), 221-227.

Garner, R. (1980). Monitoring of understanding: An investigation of good and poor readers awareness of induced miscomprehension of text. Journal of Reading Behavior, 12, 55-63.

Gutiérrez, M. (2004). Comprensión epistemológica e inferencia inductiva: un estudio sobre la física en preescolar. Revista Electrónica de Investigación Psicoeducativa, 2 (1), 63-80.

Hacker, D. (1988). Definitions and Empirical Foundations. En D. Hacker, J. Dunlosky, \& Graesser, A.
(Eds.), Metacognition in Educational Theory and Practice (pp. 1-23). London/Mahwah, New Jersey: Lawrence Erlbaum Associates Publishers.

Hacker, D. J., Plumb, C., Butterreld, E. C., Quathamer, D. \& Heineken, E. (1994). Text revision: Detection and correction of errors. Journal of Educational Psychology, 86, 65-78.

Irrazabal, N. (2007). Metacomprensión y comprensión lectora. Subjetividad y Procesos Cognitivos, 10, 46-60.

Jaramillo, A., Montaña, G. \& Rojas, L. M. (2006). Detección de errores en el proceso metacognitivo de monitoreo de la comprensión lectora en niños. Revista Latinoamericana de Ciencias Sociales, Niñez y Juventud, 4 (2) [revista electrónica].

Larigauderie, P., Gaonac'h, D. \& Lacroix, N. (1998). Working memory and error detection in texts: What are the roles of the central executive and the phonological loop? Applied Cognitive Psycho$\log y, 12,505-527$.

Latorre, D. \& Escobar, H. (2009). Relación entre la autorregulación como proceso metacognitivo y la comprensión de textos en niños de básica primaria. Tesis de maestría no publicada, Universidad Católica de Colombia, Bogotá.

Latorre, D., Navarro, C., Ramírez, N. \& Navarro, C. (2007). Estudio psicométrico de los niveles de comprensión de lectura en niños(as) de tercero de primaria. Educación y Ciencia, 10 (1), 19-32.

Levy, B. A. \& Begin, J. (1984). Proofreading familiar text: Allocating resources to perceptual and conceptual processes. Memory and Cognition, 12, 621-632.

Levy, B. A., Newell, S., Snyder, J. \& Timmins, K. (1986). Processing changes across reading encounters. Journal of Experimental Psychology: Learning, Memory and Cognition, 12, 467-478.

Matéus, G. (2007). Psicología de la comprensión textual y control de la comprensión: revisión de conceptos. Folios, 26, 39-48.

Oakhill, J. \& Yuill, N. (1991). Children's problems in text comprehension: An experimental investigation. Great Britain: Cambridge University Press.

Otero, J. (1998). Influence of knowledge activation and context on comprensión monitoring of science texts. En D. Hacker, J. Dunlosky \& A. Graesser 
(Eds.), Metacognition in Educational Theory and Practice (pp. 145-164). London/Mahwah, NJ: Lawrence Erlbaum Associates Publishers.

Otero, J. \& Campanario, J. M. (1990). Comprehension evaluation and regulation in learning from science texts. Journal of Research in Science Teaching, 27 (5), 447-460.

Piaget, J. (1977). Biología y conocimiento. Madrid: Siglo XXI.

Puche-Navarro, R. (2008). Érase una vez el desarrollo. En J. Larreamendy, R. Puche \&A. Restrepo (Eds.), Claves para pensar el cambio: ensayos sobre Psicología del desarrollo (pp. 29-69). Bogotá: Universidad de los Andes.

Reis, R. \& Spekman, N. (1983). The detection of reader-based versus text-based inconsistencies and the effects of direct training of comprehension monitoring among upper-grade poor comprehenders. Journal of Reading Behavior, 15, 49-60.

Spinillo, A. \& Da Rocha, E. (2007). Compreensão de texto em crianças: comparações entre diferentes classes de inferência a partir de una metodología on-line. Psicología Reflexao e Crítica, 20 (3), 436471.

Vergara, M. \& Velásquez, M. (1999). Relación entre la comprensión de textos escritos y la experiencia metacomprensiva. Revista Signos, 32, 149-156.

Winograd, P. \& Johnson, P. (1980). Comprehension monitoring and the error detection paradigm. Urbana, IL: University of Illinois. 